\title{
Características M aternales al Nacimiento y Destete en Cuyes de la Costa Central del Perú
}

\author{
Maternal Characteristics at Birth and Weaning in Guinea Pigs of the \\ Central Coast of Peru
Humberto Rodríguez L., ${ }^{1,2}$, Gustavo Gutiérrez R. ${ }^{\text {, }}$ Mabel Palomino T. ${ }^{1}$, Víctor Hidalgo L. ${ }^{1}$

\section{Resumen}

\section{El estudio fue llevado a cabo en la Granja de Cuyes de Cieneguilla de la Universidad} Nacional Agraria La Molina, Lima. El objetivo del estudio fue evaluar la asociación de factores fijos, como estación (E), número de parto de la madre (NP) y covariables, como peso de la madre al parto (PMP), total de nacidos (TN), número de nacidos vivos (NV) y edad al destete de las crías (EDC), con las variables total de nacidos (TN), número de nacidos vivos (NV), número de destetados (ND), peso de camada al nacimiento (PCN) y peso de camada al destete (PCD), así como estimar las correlaciones fenotípicas entre las características maternales. Se analizaron datos procedentes de 1302 camadas, recolectados entre enero de 2009 y julio de 2010. Se utilizó el procedimiento GLM del programa estadístico SAS (v. 9.1) para el análisis de variancia y la prueba de F. En el análisis para TN y NV ajustados por la covariable PMP se obtuvieron diferencias para E y NP $(\mathrm{p}<0.01)$; para ND ajustado por la covariable PMP se obtuvieron diferencias para $\mathrm{E}(\mathrm{p}<0.01)$; para PCN ajustado por la covariable PMP se hallaron diferencias para $\mathrm{E}$ y $\mathrm{NP}(\mathrm{p}<0.01)$; y para PCD ajustado por las covariables PMP, ND y EDC se obtuvieron diferencias para E, NPy $E^{*} \mathrm{NP}(\mathrm{p}<0.01)$. Las correlaciones fenotípicas estimadas fueron todas positivas entre moderadas y altas con alta significación estadística $(\mathrm{p}<0.01)$.

Palabras clave: características maternales, peso de camada al nacimiento, peso de camada al destete

\section{Abstract}

The study was carried out at the Cieneguilla guinea pig farm of La Molina National Agrarian University, Lima. The objective of the study were to evaluate the association of fixed factors such as season (E), parity (NP), and covariates such as maternal weight at

\footnotetext{
${ }^{1}$ Facultad de Zootecnia, Universidad Nacional Agraria La Molina, Lima, Perú

${ }^{2}$ E-mail: hrodri53@gmail.com
}

Recibido: 16 de junio de 2014

Aceptado: 15 de noviembre de 2014 
parity (PMP), total born (TN), number of live births (LB) and age at weaning (EDC) with total born (TN), number born alive (NV), number weaned (ND), litter weight at birth (PCN), and litter weight at weaning (PCD), and to estimate phenotypic correlations between the maternal traits. Data was collected from 1302 litters collected from January 2009 to July 2010. Analysis of variance and F tests were performed using the GLM procedure of SAS (v. 9.1). The statistical analysis for TN and NV adjusted for the covariate PMP differences were obtained for $\mathrm{E}$ and NP $(\mathrm{p}<0.01)$; for ND adjusted for the covariate PMP differences were obtained for $\mathrm{E}(\mathrm{p}<0.01)$; for PCN adjusted for the covariate PMP differences were found for $\mathrm{E}$ and $\mathrm{NP}(\mathrm{p}<0.01)$; and for PCD adjusted for covariates PMP, $\mathrm{ND}$ and EDC differences were obtained for E, NP and $E^{*} \mathrm{NP}(\mathrm{p}<0.01)$. Estimated phenotypic correlations were all positive, moderate to high, and highly significant $(\mathrm{p}<0.01)$.

Key words: maternal characteristics, litter weight at birth, litter weight at weaning

\section{INTRODUCCIÓN}

Las características maternales, como total de nacidos, número de nacidos vivos, número de destetados, peso de camada al nacimiento y peso al destete, son de importancia económica en la crianza de cuyes y deben ser considerados en los programas de mejoramiento genético. Estimados de parámetros fenotípicos y genéticos, y factores que influyen en las principales características maternales en cuyes, fueron reportados por diversos autores (Dillard et al., 1972; Chávez et al,. 1982; Rodríguez y Castro, 1982; Quijandría et al., 1983a,b,c; Chauca et al., 1984a; Solarte et al., 2002; Oblitas et al., 2006; Meza et al., 2012).

El total de nacidos es una característica maternal que está influenciada por factores como peso de la madre al parto, número de parto, estación de parto, efecto genético de líneas y sistema de apareamiento. Se han reportado valores promedios que fluctúan entre 2.0 y 3.6 crías por parto, con valores extremos de 1 a 7 crías (Rodríguez et al., 1983; Chauca et al., 1984b; Zaldívar et al., 1986; Muscari et al., 1990, 2004; Muscari et al., 1994; Dulanto, 1999; Muscari y Higaonna, 1999; Peruano, 1999).
El número de destetados es una variable influenciada por factores como habilidad materna y número de parto de la madre, estación de parto, edad al destete, tamaño de camada al nacer y mortalidad de las crías del nacimiento al destete, entre otros. Los promedios que se citan en la literatura varían entre 1.7 y 3.3 (Rodríguez et al., 1983; Chauca et al., 1984a; Aliaga et al., 1984; Aliaga y Rodríguez, 1993; Muscari et al., 1994, 2004; Dulanto, 1999; Peruano, 1999).

El peso de camada al nacimiento conjuga los pesos individuales al nacimiento y tamaño de camada al nacimiento, siendo influenciada por la habilidad materna, además de efectos de estación y número de parto de la madre. Los valores promedios fluctúan entre 220 y $490 \mathrm{~g}$ (Bocanegra y Bernal, 1982; Rodríguez et al., 1983; Aliaga et al., 1984; Aliaga y Rodríguez, 1993; Blanco y Rodríguez, 1994; Peruano, 1999).

La característica de peso de camada al destete conjuga los pesos individuales al destete y tamaño de camada al destete, y está influenciada por la habilidad materna, estación al destete, número de parto de la madre y la edad al destete de las crías. Los valores promedios fluctúan de 350 a 884 g (Rodríguez et al., 1983; Aliaga et al., 1984; Muscari et al., 1994; Peruano, 1999). 
Se han reportado correlaciones fenotípicas entre las características maternales en cuyes. Entre ellas, un valor de 0.65 con destete a 28 días para peso de camada al nacimiento y peso de camada al destete (Chávez et al., 1982), de 0.86 para número de nacidos y nacidos vivos, de 0.67 para número de nacidos y número de destetados, y de 0.78 para número de nacidos vivos y número de destetados (Quijandría et al., 1983b).

El presente estudio tuvo como objetivo evaluar características maternales al nacimiento y destete en cuyes, tales como total de nacidos (TN), número de nacidos vivos (NV), número de destetados (ND), peso de camada al nacimiento (PCN) y peso de camada al destete (PCD) por efecto de la estación (E), número de parto de la madre (NP), y covariables como peso de la madre al parto (PMP), total de nacidos (TN), número de nacidos vivos (NV) y edad al destete de las crías (EDC); así como estimar las correlaciones fenotípicas entre ellas.

\section{MateRiales y Métodos}

El estudio se desarrolló en la Granja de Cuyes «Cieneguilla», de la Universidad Nacional Agraria La Molina (UNALM), en Lima, Perú. La descripción del lugar, el origen genético de los cuyes, manejo, instalaciones y alimentación fue realizada por Rodríguez et al. (2013).

En el presente estudio, se analizó información de 1302 camadas para las variables total de nacidos (TN), número de nacidos vivos (NV), número de destetados (ND), peso de camada al nacimiento (PCN) y peso de camada al destete (PCD). Los datos fueron recolectados de enero de 2009 a julio de 2010.

Se utilizó el procedimiento GLM del programa estadístico SAS v. 9.1 para analizar las variables TN, NV, ND, PCN y PCD. El modelo incluyó como covariable el peso de la madre al parto (PMFP) para TN, NV y PCN; las covariables PMFP, NV y edad al destete de las crías (EDC) para ND; y covariables PMFP, ND y EDC para PCD. Los efectos fijos incluidos en los modelos fueron: estación $(\mathrm{E})$, número de parto de la madre (NP) y la interacción estación-número de parto $\left(\mathrm{E}^{*} \mathrm{NP}\right)$. Los efectos de las variables fueron analizados utilizando el análisis de variancia y la prueba de F.

\section{Resultados y Discusión}

\section{Tamaño de Camada al Nacimiento}

El promedio del total de nacidos por camada fue de $3.21 \pm 1.26$ (Cuadro 1). Promedios mayores fueron reportados en la línea Andina, que fue seleccionada por prolificidad en el INIA, con valores de 3.41, 3.40 y 3.35 (Chauca et al., 1984b; Dulanto, 1999; Muscari et al., 2004), en tanto que un promedio similar (3.13) fue reportado por Peruano (1999). Valores menores son reportados en la línea Perú, seleccionada por precocidad en el INIA, con valores de $2.87 \mathrm{y}$ 2.60 (Chauca et al., 1984b; Muscari et al., 1994).

El promedio de número de nacidos vivos fue de $2.92 \pm 1.20$ (Cuadro 1). Promedios cercanos fueron reportados por Chauca et al. $(1995,1997)$ con valores de 2.90 y 2.95 , respectivamente, en tanto que valores menores fueron reportados en la línea control, de apareamiento al azar $(2.65 \pm 0.86)$, línea Andina $(2.39 \pm 0.81)$ y línea Perú $(2.30 \pm$ 0.9) por Zaldívar et al. (1986) y Muscari et al. (1994), así como por Peruano (1999) de 2.67 .

En el análisis estadístico para total de nacidos y número de nacidos vivos ajustados por la covariable peso de la madre al parto, se obtuvieron diferencias significativas $(\mathrm{p}<0.01)$ para estación y número de parto, no siendo significativa la interacción E*NP. 
Cuadro 1. Características maternales de cuyes Cieneguilla en una granja de la costa central del Perú, en base a 1302 camadas

\begin{tabular}{lcccc}
\hline Variable & Promedio & $\begin{array}{c}\text { Desviación } \\
\text { estándar }\end{array}$ & Mínimo & Máximo \\
\hline Total de nacidos & 3.21 & 1.26 & 1 & 8 \\
Número de nacidos vivos & 2.92 & 1.20 & 1 & 7 \\
Número de destetados & 2.34 & 1.08 & 1 & 6 \\
Peso de camada al nacimiento (g) & 431.8 & 154.5 & 80 & 888 \\
Peso de camada al destete (g) & 691.7 & 329.4 & 88 & 1900 \\
Edad al destete de las crías (días) & 14.2 & 3.4 & 6 & 21 \\
Peso de la madre al parto (g) & 1534.4 & 252.8 & 618 & 2415 \\
\hline
\end{tabular}

Cuadro 2. Promedios de total de nacidos y de nacidos vivos, y número de destetados de cuyes Cieneguilla en una granja de la costa central del Perú, según la estación de parto

\begin{tabular}{lcccc}
\hline Estación & N. & $\begin{array}{c}\text { Total de } \\
\text { nacidos } \\
\bar{x} \pm \text { D.E. }\end{array}$ & $\begin{array}{c}\text { Nacidos vivos } \\
x \pm \text { D.E. }\end{array}$ & $\begin{array}{c}\text { N. }^{\circ} \text { de } \\
\text { destetados } \\
\bar{x} \pm \text { D.E. }\end{array}$ \\
\hline Primavera & 354 & $3.21 \pm 1.27^{\mathrm{b}}$ & $2.90 \pm 1.21^{\mathrm{b}}$ & $2.16 \pm 1.07^{\mathrm{b}}$ \\
Verano & 390 & $3.05 \pm 1.22^{\mathrm{c}}$ & $2.69 \pm 1.13^{\mathrm{c}}$ & $2.23 \pm 1.01^{\mathrm{b}}$ \\
Otoño & 295 & $3.14 \pm 1.20^{\mathrm{bc}}$ & $2.93 \pm 1.16^{\mathrm{b}}$ & $2.43 \pm 1.08^{\mathrm{a}}$ \\
Invierno & 262 & $3.54 \pm 1.30^{\mathrm{a}}$ & $3.29 \pm 1.24^{\mathrm{a}}$ & $2.62 \pm 1.16^{\mathrm{a}}$ \\
\hline
\end{tabular}

${ }^{a, b}$ Superíndices diferentes dentro de cada columna indican diferencia estadística $(p \varangle 0.05)$

Los partos ocurridos en invierno presentaron mayores valores de $\mathrm{TN}$ y NV respecto a las demás estaciones. A su vez, los partos ocurridos en primavera y otoño presentaron valores similares para ambas características y superiores a los partos de verano (Cuadro 2). Chauca et al. (1995) reportan tamaño de camada al nacer de 2.51 en otoño y 2.90 en invierno; valores menores que los del estudio.

El valor promedio de $\mathrm{TN}$ al primer parto fue inferior a los demás partos
(Cuadro 3). En contraste, para NV solo se hallaron diferencias entre el primer y sexto parto $(\mathrm{p}<0.05)$. Peruano (1999) reporta valores similares de tamaño de camada al nacer (3.13), en tanto que Muscari et al. (2004) mencionan que el número de parto influye sobre el tamaño de camada, habiendo un incremento del primer al segundo parto. En relación con NV, Peruano (1999) halló valores crecientes del primer (2.84) al cuarto parto (3.12) en la línea Perú, con diferencias significativas entre los partos 3 y 4 respecto a los partos 1 y 2 . 
Cuadro 3. Promedios de total de nacidos y nacidos vivos de cuyes Cieneguilla en una granja de la costa central del Perú, según el número de parto

\begin{tabular}{cccc}
\hline Número de parto & N. $^{\circ}$ & $\begin{array}{c}\text { Total de nacidos } \\
\bar{x} \pm \text { D.E. }\end{array}$ & $\begin{array}{c}\text { Nacidos vivos } \\
\bar{x} \pm \text { D.E. }\end{array}$ \\
\hline 1 & 296 & $3.13 \pm 1.15^{\mathrm{b}}$ & $2.78 \pm 1.09^{\mathrm{b}}$ \\
2 & 366 & $3.17 \pm 1.21^{\mathrm{a}}$ & $2.89 \pm 1.11^{\mathrm{ab}}$ \\
3 & 302 & $3.23 \pm 1.33^{\mathrm{a}}$ & $2.99 \pm 1.30^{\mathrm{ab}}$ \\
4 & 217 & $3.28 \pm 1.36^{\mathrm{a}}$ & $2.99 \pm 1.32^{\mathrm{ab}}$ \\
5 & 94 & $3.34 \pm 1.27^{\mathrm{a}}$ & $3.05 \pm 1.19^{\mathrm{ab}}$ \\
6 & 26 & $3.46 \pm 1.39^{\mathrm{a}}$ & $3.27 \pm 1.15^{\mathrm{a}}$ \\
\hline
\end{tabular}

${ }^{a, b}$ Superíndices diferentes dentro de cada columna indican diferencia estadística $(p \varangle 0.05$ )

Cuadro 4. Promedios de peso de camada al nacimiento y al destete de cuyes Cieneguilla en una granja de la costa central del Perú, según estación de parto

\begin{tabular}{lccc}
\hline Estación & N. & $\begin{array}{c}\text { Peso de camada al } \\
\text { nacimiento }(\mathrm{g}) \\
\bar{x} \pm \mathrm{D} . \mathrm{E} .\end{array}$ & $\begin{array}{c}\text { Peso de camada al } \\
\text { destete }(\mathrm{g}) \\
\bar{x} \pm \mathrm{D} . \mathrm{E} .\end{array}$ \\
\hline Primavera & 354 & $395.5 \pm 139.8^{\mathrm{c}}$ & $605.5 \pm 303.3^{\mathrm{d}}$ \\
Verano & 390 & $409.2 \pm 147.2^{\mathrm{c}}$ & $657.8 \pm 303.5^{\mathrm{c}}$ \\
Otoño & 295 & $440.0 \pm 152.8^{\mathrm{b}}$ & $750.7 \pm 343.9^{\mathrm{b}}$ \\
Invierno & 262 & $505.5 \pm 160.7^{\mathrm{a}}$ & $792.3 \pm 346.6^{\mathrm{a}}$ \\
\hline
\end{tabular}

${ }^{a, b}$ Superíndices diferentes dentro de cada columna indican diferencia estadística $(p \varangle 0.05)$

\section{Peso de Camada al Nacimiento}

El valor promedio de PCN fue de $431.8 \pm 154.5 \mathrm{~g}$ (Cuadro 1). Promedios mayores $(451.5 \mathrm{~g})$ fueron reportados por Peruano (1999), y promedios menores $(352.9 \mathrm{~g})$ fueron reportados por Chauca $e t$ al. (1995). Los promedios de PCN ajustado por la covariable peso de la madre al parto fueron altamente significativos $(\mathrm{p}<0.01)$ para la estación y número de parto.

Los valores de PCN para las nacidas en invierno fueron superiores a los valores de las demás estaciones, seguida del OCN en otoño. Asimismo, no hubo diferencias entre verano y primavera (Cuadro 4). Por otro lado, Chauca et al. (1995) encontraron que el peso total de camada al nacimiento fue mayor a medida que se incrementaba el número de crías nacidas.

Según el número de parto, los cuyes de quinto parto presentaron los mayores valores de PCN, en tanto que los cuyes del primer y segundo parto presentaron los menores pesos (Cuadro 5). Respecto al número de parto, Peruano (1999) halló que 
Cuadro 5. Promedios de peso de camada al nacimiento y destete de cuyes Cieneguilla en una granja de la costa central del Perú, según el número de parto

\begin{tabular}{cccc}
\hline Número de parto & N. & $\begin{array}{c}\text { Peso de camada al } \\
\text { nacimiento }(\mathrm{g}) \\
\bar{x} \pm \text { D.E. }\end{array}$ & $\begin{array}{c}\text { Peso de camada al } \\
\text { destete }(\mathrm{g}) \\
\bar{x} \pm \text { D.E. }\end{array}$ \\
\hline 1 & 296 & $395.2 \pm 138.1^{\mathrm{c}}$ & $582.5 \pm 265.6^{\mathrm{c}}$ \\
2 & 366 & $415.1 \pm 135.3^{\mathrm{c}}$ & $665.8 \pm 300.5^{\mathrm{ab}}$ \\
3 & 302 & $458.2 \pm 166.8^{\mathrm{b}}$ & $753.4 \pm 332.8^{\mathrm{a}}$ \\
4 & 217 & $448.7 \pm 164.4^{\mathrm{b}}$ & $745.8 \pm 372.4^{\mathrm{a}}$ \\
5 & 94 & $478.4 \pm 176.4^{\mathrm{a}}$ & $785.2 \pm 395.4^{\mathrm{a}}$ \\
6 & 26 & $469.2 \pm 156.1^{\mathrm{ab}}$ & $792.5 \pm 342.3^{\mathrm{a}}$ \\
\hline
\end{tabular}

${ }^{a, b}$ Superíndices diferentes dentro de cada columna indican diferencia estadística $(p \varangle 0.05$ )

Cuadro 6. Correlaciones fenotípicas entre las variables respuesta ${ }^{1}$ en cuyes Cieneguilla en una granja de la costa central del Perú

\begin{tabular}{|c|c|c|c|c|c|}
\hline & $\mathrm{TN}$ & $\mathrm{NV}$ & $\mathrm{PCN}$ & ND & PCD \\
\hline $\mathrm{TN}$ & 1.0 & $0.86 * *$ & $0.67 * *$ & $0.55^{* *}$ & $0.31 * *$ \\
\hline $\mathrm{NV}$ & & 1.0 & $0.86 * *$ & $0.67 * *$ & $0.45^{* *}$ \\
\hline $\mathrm{PCN}$ & & & 1.0 & $0.67 * *$ & $0.82 * *$ \\
\hline ND & & & & 1.0 & $0.62 * *$ \\
\hline PCD & & & & & 1.0 \\
\hline
\end{tabular}

el mayor y menor peso al cuarto y primer parto, respectivamente, manteniendo la misma tendencia que la del presente estudio.

\section{Número de Destetados}

El ND promedio fue de $2.34 \pm 1.08$ (Cuadro 1), el cual se explica por la alta mortalidad ocurrida entre el nacimiento y destete de las crías. Promedios mayores han sido reportados por Aliaga et al. (1984), de 3.08, Chauca et al. (1997) de 2.84, y Peruano (1999) de 2.67; mientras que promedios similares son reportados por Muscari et al. (1994) de 2.00y por Dulanto (1999) de 2.24, ambos en la línea Perú. El ND ajustado por la covariable peso de la madre al parto fue afectado por la estación $(\mathrm{p}<0.01)$; sin embargo, no fue afectado por el número de parto ni por la interacción E*NP. 


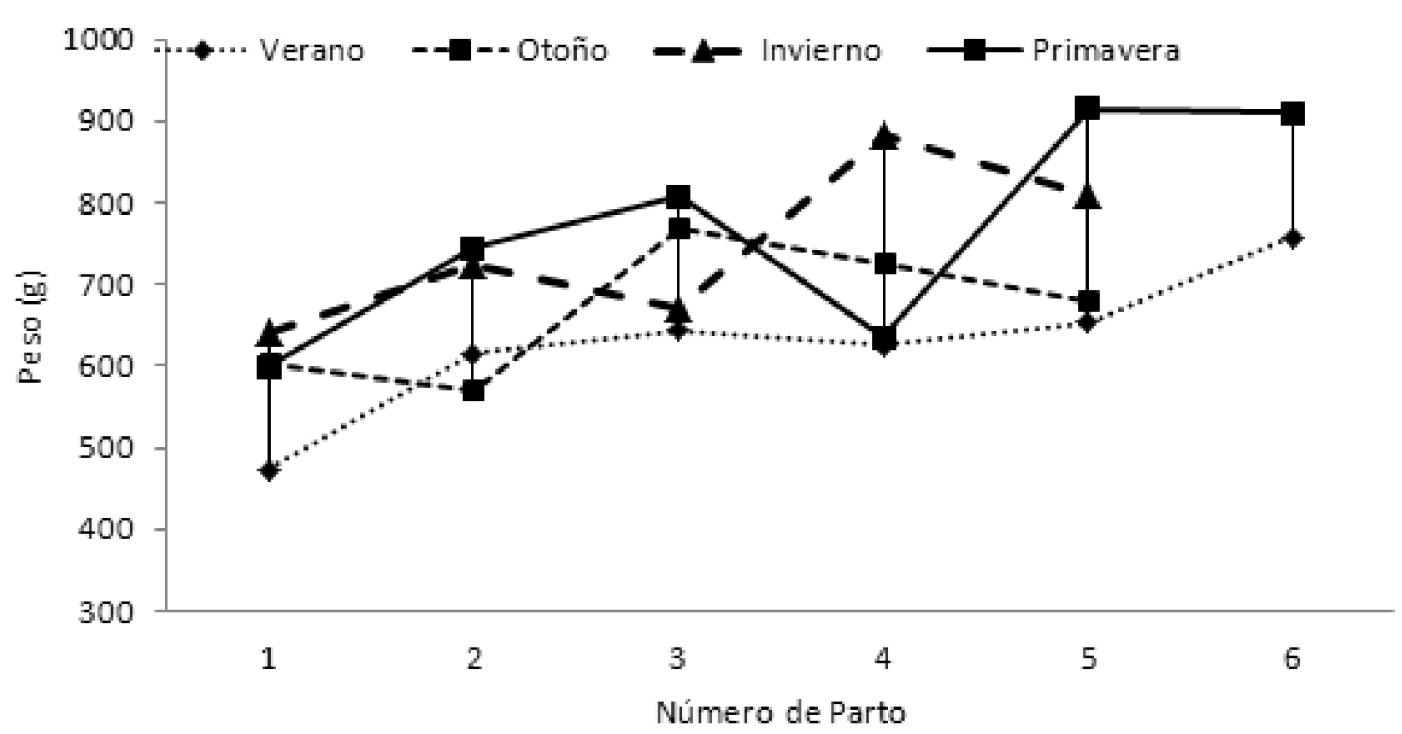

Figura 1. Interacción de estación del año x número de parto para la variable peso de camada al destete en cuyes Cieneguilla en una granja de la costa central del Perú

Si bien el número de cuyes destetados en invierno y otoño son similares y superiores a los de verano y primavera (Cuadro 2), no se pudo comparar con otros resultados al no hallarse referencias bibliográficas sobre el efecto de la estación de parto sobre el número de destetados.

\section{Peso de Camada al Destete}

El promedio de peso de camada al destete fue de $691.7 \pm 329.4 \mathrm{~g}$ (Cuadro 1). Este valor es menor que los $825.3 \mathrm{~g}$ reportado por Peruano (1999), similar a los 658.5 y $668.0 \mathrm{~g}$ reportados por Zaldívar $e t$ al. (1986) y Chauca et al. (1995), respectivamente, quienes afirman que el peso total de camada al destete fue mayor a medida que se incrementaba el número de destetados. Asimismo, fue mayor de los 524.0 g reportados por Muscari et al. (1994) en la línea Perú. Al análisis estadístico del peso de camada al destete ajustados por las covariables peso de la madre al parto, número de destetados y edad al destete de las crías, se obtuvo diferencias significativas $(\mathrm{p}<0.01)$ para estación, número de parto y la interacción $E^{*}$ NP.

Si bien los mayores pesos de camada se observaron en invierno (Cuadro 4) y en hembras del tercer al sexto parto (Cuadro5), no se pudo comparar con otros resultados al no hallarse referencias bibliográficas sobre los efectos de la estación y del número de parto sobre el peso de camada al destete.

En la interacción $\mathrm{E}^{*} \mathrm{NP}$, las diferencias estadísticas $(p<0.01)$ se deben fundamentalmente al número de parto por las fluctuaciones en la variable y manteniéndose las tendencias respecto a la asociación del peso de camada al destete con las estaciones (Fig. 1).

\section{Correlaciones Fenotípicas}

Las correlaciones fenotípicas estimadas entre las características TN, NV, ND, PCN, y PCD fueron positivas $(\mathrm{p}<0.01)$, variando de moderadas a altas (Cuadro 6). 
La correlación fenotípica del total de nacidos y número de nacidos vivos fue de 0.86. Al respecto Quijandría et al. (1983b) reportaron un valor similar de 0.86 , mientras que para el total de nacidos y número de destetados fue de 0.55. Quijandría et al. (1983b) reportaron un valor de 0.67 , superior al del presente estudio. No existen referencias bibliográficas para las demás correlaciones fenotípicas.

\section{Conclusiones}

- Para el total de nacidos, número de nacidos vivos y peso de camada al nacimiento, ajustado por la covariable peso de la madre al parto, se obtuvieron diferencias asociadas a la estación y número de parto de la madre $(\mathrm{p}<0.01)$; donde los mejores promedios fueron en el invierno, así como aumentan gradualmente del primer al sexto parto.

- Para el número de destetados ajustados por la covariable peso de las madres al parto, se obtuvieron diferencias asociadas a la estación ( $\mathrm{p}<0.01)$, donde el número de cuyes destetados en invierno y otoño fue superior al número de destetados en verano y primavera.

- Para el peso de camada al destete ajustado por las covariables peso de la madre al parto, número de destetados y edad al destete de las crías, se obtuvieron diferencias asociados a la estación, número de parto y la interacción entre ambas ( $p<0.01$ ), donde los pesos de camada al destete en invierno fueron superiores a las demás estaciones, así como aquellos del tercer al sexto parto fueron superiores al primer y segundo parto.

- Las correlaciones fenotípicas entre las características maternales fueron positivas entre moderadas y altas $(\mathrm{p}<0.01)$.

\section{Agradecimientos}

Los autores agradecen al Programa de Investigación y Proyección Social en Carnes de la Facultad de Zootecnia de la Universi- dad Nacional Agraria La Molina, que mediante la Granja de Cuyes de Cieneguilla y de su administradora, la Ing. Teresa Montes, brindaron las facilidades y el financiamiento para la toma y recolección de datos. Del mismo modo, se agradece al personal administrativo y obrero por el apoyo brindado.

\section{Literatura Citada}

1. Aliaga L, Rodríguez H, Borja A, Núñez E. 1984. Sistema de empadre y flushing proteico en cuyes. Resúmenes VII Reunión APPA. Lima, Perú.

2. Aliaga F, Rodríguez H. 1993. Cruzamiento de cuyes hembras criollas con machos mejorados en el distrito de Sicaya, Junín. Resúmenes XVI Reunión APPA. Piura. Perú.

3. Blanco P, Rodríguez H. 1994. Cruzamiento simple con tres líneas consanguíneas de cuyes. Resúmenes XVII Reunión APPA. Lima. Perú.

4. Bocanegra C, Bernal S. 1982. Productividad del cuy hembra al primer parto bajo tres niveles de gallinaza. Resúmenes V Reunión APPA. Cajamarca, Perú.

5. Chauca L, Augustín R, Muscari J, Zaldivar M. 1984a. Determinación de la edad óptima de destete en cuyes. Resúmenes VII Reunión APPA. Lima, Perú.

6. Chauca L, Quijandría B, Saravia J, Muscari J. 1984b. Evaluación de la tasa de crecimiento, tamaño de camada y conversión alimenticia de cuatro líneas de cuyes. Resúmenes VII Reunión APPA. Lima, Perú.

7. Chauca L, Muscari J, Ordoñez R, Higaonna R. 1995. Efecto del tamaño de camada sobre la performance de cuyes en lactación. Resúmenes XVIII Reunión APPA. Lambayeque. Perú.

8. Chauca L, Peruano D, Muscari J. 1997. Comportamiento reproductivo de gestaciones postpartum y postdestete en cuyes (Cavia porcellus) manejados en empadre continuo durante un año. Re- 
súmenes XX Reunión APPA. Tingo María. Perú.

9. Chávez J, Muscari J, Arévalo J. 1982. Parámetros genéticos del peso de camada al nacimiento y al destete en cuyes (Cavia porcellus). Resúmenes V Reunión APPA. Cajamarca, Perú.

10. Dillard EU, Vaccaro $R$, Lozano $J$, Robison OW. 1972. Phenotypic and genetic parameters for growth in guinea pigs. J Anim Sci 34: 193-195.

11. Dulanto M. 1999. Parámetros reproductivos y productivos de tres líneas puras y dos grados de cruzamiento entre líneas de cuyes. Tesis de Ing. Zootecnista. Lima: Univ Nacional Agraria La Molina. 69 p.

12. Meza E, Raymondi J, Cisneros $S$. 2012. Evaluación genética de un plantel de cuyes reproductores de genotipo Perú. Resúmenes XXXV Reunión APPA. Trujillo. Perú.

13. Muscari J, Zaldivar M, Chauca L. 1990. Evaluación de la prolificidad de una línea genética de cuyes. Resúmenes XII Reunión ALPA. Campinas, SP, Brasil.

14. Muscari J, Chauca L, Higaonna R. 1994. Caracterización de una línea mejorada de cuyes: Perú. Resúmenes XVII Reunión APPA. Lima, Perú.

15. Muscari J, Higaonna R. 1999. Comportamiento reproductivo de las líneas de cuyes Perú, Inti y Andina. Resúmenes XXII Reunión APPA. Huancavelica, Perú.

16. Muscari J, Chauca L, Higaonna R. 2004. Caracterización de la línea de cuyes Andina. Resúmenes XXVII Reunión APPA. Piura, Perú.

17. Oblitas J, Oscanoa W, Kajjak N. 2006. Parámetros genéticos en cuyes de la Estación Experimental INIEA Huancayo. Resúmenes XXIX Reunión APPA. Huancayo, Perú.
18. Peruano D. 1999. Evaluación de la vida productiva y reproductiva del cuy hembra (Cavia porcellus) en cuatro partos utilizando el empadre continuo. Tesis de Ing. Zootecnista. Lima: Univ Nacional Agraria La Molina. 71 p.

19. Quijandría B, Chauca L, Robison $O W$. 1983a. Selection in guinea pigs: I. Estimation of phenotypic and genetic parameters for litter size and body weight. J Anim Sci 56: 814-819.

20. Quijandría B, Zaldivar M, Robison $O W .1983 b$. Selection in guinea pigs: II. Direct response for litter size and body weight. J Anim Sci 56: 820-828.

21. Quijandría B, Muscari J, Robinson $O W .1983 c$. Selection in guinea pigs: III. Correlated responses to selection for litter size and body weight. J Anim Sci 56: 829832.

22. Rodríguez H, Castro C. 1982. Parámetros genéticos en cuyes. Resúmenes V Reunión APPA. Cajamarca, Perú.

23. Rodríguez H, Aliaga L, Borja A, Veliz V. 1983. Empadre a 60, 75 y 90 días en cuyes. Resúmenes VI Reunión APPA. Lambayeque, Perú.

24. Rodríguez H, Palomino M, Hidalgo V, Gutiérrez G. 2013. Efectos de factores fijos y al azar sobre el peso al nacimiento y al destete en cuyes de la costa central. Rev Inv Vet Perú 24: 16-24.

25. Solarte C, Imuez AM, Pérez T. 2002. Modelo animal multicarácter para la estimación de parámetros genéticos del Cavia porcellus en Colombia. Rev Cub Cienc Agríc 36(1): 19-24.

26. Zaldivar M, Chauca L, Quijandría B, Poma Z. 1986. Estudio comparativo de la producción de cuatro líneas de cuyes (Cavia porcellus) generación F11. Resúmenes IX Reunión APPA. Tingo María, Perú. 